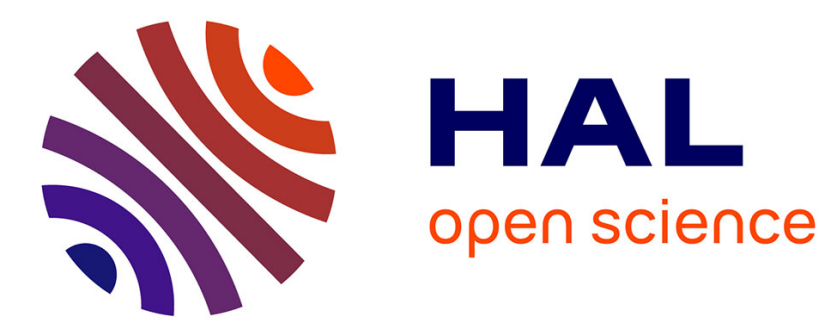

\title{
Situation assessment: an end-to-end process for the detection of objects of interest
}

\author{
Evangeline Pollard, Michèle Rombaut, Benjamin Pannetier
}

\section{To cite this version:}

Evangeline Pollard, Michèle Rombaut, Benjamin Pannetier. Situation assessment: an end-to-end process for the detection of objects of interest. IEEE Transactions on Aerospace and Electronic Systems, 2013, 49 (4), pp.2195-2210. 10.1109/TAES.2013.6621810 . hal-00763715v2

\section{HAL Id: hal-00763715 https://hal.inria.fr/hal-00763715v2}

Submitted on 8 Jan 2014

HAL is a multi-disciplinary open access archive for the deposit and dissemination of scientific research documents, whether they are published or not. The documents may come from teaching and research institutions in France or abroad, or from public or private research centers.
L'archive ouverte pluridisciplinaire HAL, est destinée au dépôt et à la diffusion de documents scientifiques de niveau recherche, publiés ou non, émanant des établissements d'enseignement et de recherche français ou étrangers, des laboratoires publics ou privés. 


\section{Situation Assessment: An End-to-End Process for the Detection of Objects of}

\section{Interest}

\section{EVANGELINE POLLARD}

French National Institute for Research in

Computer Science and Control (INRIA)

\section{MICHÈLE ROMBAUT}

Grenoble Image Parole Signal Automatique

Laboratory (GIPSA-Lab)

France

BENJAMIN PANNETIER

French Aerospace Laboratory (ONERA)

Semiautomatic approaches are developed for wide area situation assessment in near-real-time. The two-step method consists of two granularity levels. The first entity assessment uses a new multi-target tracking (MTT) algorithm (hybridization of Gaussian mixture-Cardinalized probability hypothesis density (GM-CPHD) filter and multiple hypothesis tracker (MHT) with road constraints) on ground moving target indicator (GMTI) data. The situation is then assessed by detecting objects of interest such as convoys with other data types (synthetic aperture radar (SAR), video). These detections are based on Bayesian networks and their credibilistic counterpart.

Manuscript received November 15, 2011; revised June 19 and August 27, 2012; released for publication November 26, 2012.

IEEE Log No. T-AES/49/4/944694.

Refereeing of this contribution was handled by W. Koch.

Authors' addresses: E. Pollard, INRIA, IMARA, Domaine de Voluceau-Rocquencourt, B. P. 105, Le Chesnay, 78153, France, E-mail: (evangeline.pollard@cpe.fr); M. Rombaut, Department of Image and Signal (DIS), Grenoble Image Parole Signal Automatique Laboratory (GIPSA-lab), Grenoble, France; B. Pannetier, Department of Modeling and Information Processing, ONERA, French Aerospace Lab, F-91761, Palaiseau, France.

0018-9251/13/\$26.00 (c) 2013 IEEE

\section{INTRODUCTION}

Information processing is a challenging goal for any automatic system. The increasing number of sensors involves the use of data fusion techniques in order to raise the semantic level of a piece of information, i.e., transform a numerical value into meaningful information and produce relevant information regarding a specific problem.

In the battlefield surveillance domain, data are produced by heterogeneous sensors like airborne ground moving target indicator (GMTI) data, synthetic aperture radar (SAR) images, or video coming from unmanned aerial vehicles (UAV). Data can also come from databases contained in the Geographical Information System (GIS). In the situation assessment context, the goal is to refine the quality of information and detect objects of interest from an operational point of view. In this application we decided to first focus on the object-of-interest "convoy" which is defined as an aggregate of vehicles with a particular kinematic behavior. By considering its strategic purposes (moving of troops or equipment), this object is interesting but difficult to track and to estimate. Our idea is the following challenging task: to develop a method for detecting and evaluating a convoy in order to generalize it to any object of interest. We want to highlight the fact that we consider an asymmetric conflict context, and we want to detect convoys (and more generally objects of interest) in the midst of civilian traffic. The purpose of this article is to summarize the entire process for convoy detection in the midst of civilian traffic and to test the limit of our algorithm by evaluating the performances based on complex scenarios.

Traditionally, the preamble of this operation is to make a global evaluation of the situation by detecting and characterizing entities [1] (level 1 of the Joint Directors of Laboratories (JDL) model called the entity assessment). As GMTI sensors cover wide surveillance areas and are able to detect moving targets by measuring their Doppler shift, they are traditionally used during the multi-target tracking (MTT) step [2]. Then, in order to specify entity behavior and detect objects of interest, a more refined assessment (level 2 of the JDL model which is called the situation assessment) can be done by using other data types like SAR or video. As described in Fig. 1, our proposed approach has two steps.

The first step consists of developing an MTT algorithm able to deal with many classical tracking problems such as false alarms and nondetection, as well as closely spaced targets. In some previous work [3] we developed a new MTT algorithm which grappled with these problems in order to obtain an image as close to the actual ground picture as possible and to construct realistic scenarios. 


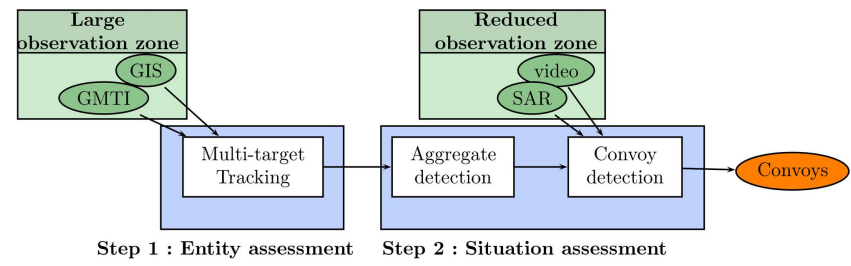

Fig. 1. Convoy detection process. First step consists of the MTT algorithm, second step concerns detection and characterization of convoys.

During step two represented in Fig. 1, we use tracks coming from the MTT algorithm, in addition to SAR and video information, in order to detect convoys. In some previous work we developed a method based on graphical models from a probabilistic approach [4] as well as from a credibilistic approach [5].

The goal of this paper is to summarize the entire process, present some improvements, and develop the estimation of the convoy state. An estimation of the number of targets belonging to the convoy is provided and tested on two challenging scenarios. In the first scenario the algorithm is confronted with a slowing down of traffic, creating an aggregate of vehicles. This scenario is intended to show our approach's robustness to false alarms. In the second scenario a convoy is separating into two smaller convoys. This is intended to show the robustness of our approach to complex maneuvers.

The paper is organized as follows: Section II is a theoretical description of the developed MTT algorithm and contains two main subsections: the labeled Gaussian mixture-Cardinalized probability hypothesis density (GM-CPHD) and the hybridization. Section III describes our approach to the convoy detection: one subsection is about the probabilistic approach, whereas a second subsection describes the credibilistic one. Finally, Section IV describes the simulations and performances of the proposed system before we conclude in Section V.

\section{MULTI-TARGET TRACKING}

The MTT algorithm must be able to deal with many classical tracking problems like:

1) Data relevance: a large number of measurements due to the large surveillance area, containing false alarms and suffering from nondetection, due to ground environment and radar cross section (RCS) of ground targets.

2) Manoeuvring targets: targets can completely change their trajectory between two data sets, and the motion model does not match anymore with the true target dynamics.

3) Birth and death targets: this problem implies the use of a very competitive target detection process in the tracking algorithm.
4) Closely spaced targets: targets which are close to each other can generate unresolved targets and make the problem of data association more complex.

In this section we describe the new MTT developed for this application. First, in Section II-A, we define the measurement and motion model of one target. In Section II-B we analyze the MTT issues in a GMTI context. Finally, in Sections II-C and II-D, we review principles of the new proposed MTT algorithm.

\section{A. Measurement and Motion Model}

During a surveillance operation a local Cartesian reference frame is defined by operators. The state $x_{k, j}$ of one target $j$ is defined at each iteration $k$ in this frame by its position $\left(\mathrm{x}_{k, j}, \mathrm{y}_{k, j}\right)$ and its velocity $\left(\dot{\mathrm{x}}_{k, j}, \dot{\mathrm{y}}_{k, j}\right)$ as

$$
x_{k, j}=\left[\mathrm{x}_{k, j}, \dot{\mathrm{x}}_{k, j}, \mathrm{y}_{k, j}, \dot{\mathrm{y}}_{k, j}\right]^{T} .
$$

Data used for observing targets come from a GMTI sensor by measuring their Doppler shift. Each sensor gets a measurement vector $Z_{k}=\left\{z_{k, 1}, \ldots, z_{k, m_{k}}\right\}$ at each iteration $k$ composed of $m_{k}$ measurements. Each measurement $z_{k, i}$, issued from a target $j$, corresponds to the observed position vector and is given by

$$
z_{k, i}=H \cdot x_{k, j}+b_{k}
$$

where $H$ is the transforming matrix from state space to measurement space and $b_{k}$ is a white Gaussian noise with a known covariance matrix $R_{k}=\mathbb{E}\left[b_{k} \cdot b_{k}^{T}\right]$ as defined in [6].

By examining the state of one target $x_{k, j}$, the state equation is here limited to linear cases:

$$
x_{k+1, j}=F_{k, l} \cdot x_{k, j}+\Gamma_{k} \cdot \nu_{k, l}
$$

where $F_{k, l}$ is the state transition matrix according to the model $l$ and at iteration $k$ and $\nu_{k, l}$ is a zero-mean, white Gaussian process noise with the known covariance matrix $Q_{l}$ which models the target acceleration:

$$
Q_{l}=\mathbb{E}\left[\nu_{k, l} \cdot \nu_{k, l}^{T}\right]=q_{l} \Gamma_{k} \cdot \Gamma_{k}^{T}
$$

with $q_{l}$ being the noise considered with model $l$ and $\Gamma_{k}$ as defined by Bar-Shalom in [6].

In addition to the state estimation $\hat{x}_{k, j}$, we want to produce an estimation $P_{k, j}$ of its uncertainty covariance associated with the target state estimation for each target $j$ at iteration $k$.

Targets are detected with a detection probability denoted as $P_{\mathrm{d}}$. The measurement set contains false alarms of density $\beta_{\mathrm{fa}}$. This density is uniform, and its cardinality $N_{\mathrm{fa}}$ is assumed to follow a Poisson process on the observed area noted $V_{k}$ as:

$$
N_{\mathrm{fa}}=\beta_{\mathrm{fa}} \cdot V_{k} .
$$




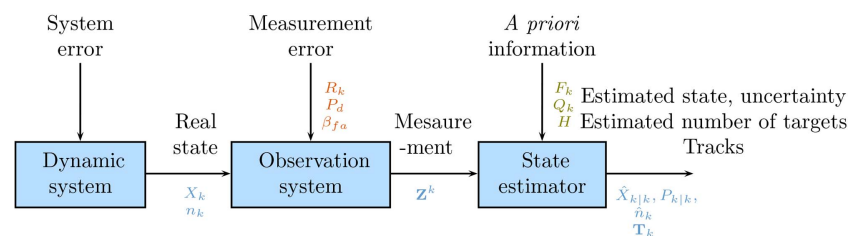

Fig. 2. Schematic view of estimation problem in multi-object context.

\section{B. Multi-Target Tracking Issues}

MTT is a well-studied problem [7]. In this context tracking issues are multiple. The problem is to estimate a number of characteristics describing the scene, which are the following.

1) The multi-target state $X_{k}=\left\{x_{k, 1}, \ldots, x_{k, n_{k}}\right\}$,

2) the tracks associated to targets, and

$3)$ the number of targets $n_{k}$ at each time.

The state estimation for a variable number $n_{k}$ of targets at each iteration $k$ is defined as

$$
\hat{X}_{k \mid k}=\left\{\hat{x}_{k, j}\right\}_{j=1}^{\hat{n}_{k}} .
$$

A track $\mathcal{T}_{k, p}$ is defined at one iteration $k$ as a sequence of states describing the trajectory of one target $p$. Mathematically, a track is defined as

$$
\mathcal{T}_{k, p}=\left\{\hat{x}_{k, j}, P_{k, j}, s_{k, p}, \mathcal{T}_{k-1, p}\right\}
$$

where $s_{k, p}$ is the score associated with $p$, which can be seen as a measure for its reliability. In an operational context it is necessary to label targets and to produce at each iteration $k$ a set of tracks $\mathcal{T}_{k}$.

By considering the birth and death target process, the system produces an estimation $\hat{n}_{k}$ of the number of targets at each iteration $k$. The accumulated tracks from the start iteration to iteration $k$ are noted $\mathbf{T}_{k}$.

By considering Section II-A and the current subsection, the MTT issues can be summarized by Fig. 2, inspired from [6]. In order to estimate the characteristics and knowing that the state space is continuous, the following two approaches are conceivable.

1) Discretize the state space and consider the case of each target $x_{k, j}$ individually [8]. This implies a systematic target-to-measurement association, like with the MHT (multiple hypothesis tracker), where all hypotheses for a measurement (be it a false alarm, a new track, or the continuity of existing tracks) are considered.

2) Consider the multi-target state $X_{k}$ as a multi-modal variable, by considering $X_{k}$ a random finite set (RFS) [9] that avoids the problem of data association. Some closed-form solutions are actually available like the GM-CPHD.

In the next subsection a labeling step is proposed for the GM-CPHD filter to make it compatible with the MTT issues presented in this section and more specifically in order to provide tracks describing target dynamics. In Section II-D the two algorithms are compared, and finally, an original hybridizing approach is proposed, which is equally efficient for well-separated as well as closely-spaced targets.

\section{Labeled GM-CPHD Filter}

The cardinalized probability hypothesis density (CPHD) filter was first introduced by Mahler in [10], [11]. It is a generalization of the previous probability hypothesis density (PHD) filter, mainly presented in [12], [9]. The CPHD filter is originally a nonlinear/non-Gaussian filter. However, under linear and Gaussian assumptions, closed-form equations can be implemented. In this work we focus on the Gaussian mixture recursion (GM-CPHD filter) under the assumption of linear Gaussian dynamics and the state independence of the detection and survival probability [13] (we do not deal with a Monte-Carlo implementation [14] which is very time consuming in a real multi-target context).

The GM-CPHD filter is based on the study of the joint probability density of the RFS describing target dynamics and measurements. The first-order moment of this RFS, called the intensity function $v$, is the function whose integral in any region of the state space yields the expected number of targets in that region. Points with the highest density are then the expected targets. At each iteration $k$, in addition to the intensity function $v_{k}$, it propagates the entire probability distribution of the number of targets, noted $p_{k}$.

To summarize, at each iteration, the classical GM-CPHD provides a Gaussian set $v_{k}$ describing the first moment of the joint probability density function of the target set $X_{k}$, written as

$$
v_{k}(x)=\sum_{i=1}^{N_{k}^{\mathcal{G}}} w_{k, i} \cdot \mathcal{N}\left(x ; m_{k, i}, P_{k, i}\right)
$$

where $\mathcal{N}\left(x ; m_{k, i}, P_{k, i}\right)$ denotes the Gaussian density with parameters $w_{k, i}, m_{k, i}$, and $P_{k, i}$ being the weight, the mean, and the covariance, and $N_{k}^{\mathcal{G}}$ is the number of Gaussian components.

By considering the probability distribution of the number of targets $p_{k}$, it provides an estimation of the number of targets $\hat{n}_{k} \in \mathbf{R}^{+}$, written as

$$
\hat{n}_{k}=\sum_{n=1}^{\infty} n \cdot p_{k}(n) .
$$

For the track labeling the number of targets $\hat{N}_{k}^{\star} \in$ $\mathbf{N}^{+}$is considered

$$
\hat{N}_{k}^{\star}=\underset{n \in\left\{1, \ldots, N_{\max }\right\}}{\arg \max } p_{k}(n)
$$

where $N_{\max }$ is the maximum number of considered targets.

In order to summarize the global state of the scene as defined in Section II-B, and especially the 
definition of tracks, it is necessary to label targets at any time. In the classical version of the GM-CPHD filter, the problem of track labeling is not considered. Some authors study this problem [15]. For example, Clark, et al., in [16], proposed that the GM-PHD filter assign a tag to each Gaussian component and keep as tracks Gaussians with weights above a certain threshold. However, when a measurement is not received, the weight drops below the desired threshold but the Gaussian component is not deleted and the target trajectory will be specified a posteriori after the weight is again above the desired threshold. More recently Erdinc, et al. [17] propose an approach for the GM-CPHD filter labeling based on Panta's work [18]. But these approaches do not take advantage of the estimated number of targets $\hat{N}_{k}^{\star}$.

The goal of labeling is to provide identities to targets by selecting tracks among the set $\mathcal{G}_{k}$ at iteration $k$ of Gaussian components $\mathcal{G}_{k, n}=$ $\mathcal{N}\left(x ; m_{k, n}, P_{k, n}\right)$. We propose to formalize the problem as the calculation of the best association matrix $\mathbf{A}_{k}$ between a Gaussian set $\mathcal{G}_{k}$ and a set of predicted track $\mathcal{T}_{k}=\left\{\mathcal{T}_{k \mid k-1, m}\right\}_{\forall m \in\left\{1, \ldots, \hat{N}_{k-1}^{\star}\right\}}$ at each iteration $k$.

In order to limit the time computation, the matrix of feasible association $\tilde{A}_{k}$ is first calculated by using a gating test [19]:

$$
\begin{aligned}
& \tilde{A}_{k}(m, n)= \\
& \begin{cases}1 & \text { if } \mathcal{G}_{k, n} \text { is statistically closed to } \mathcal{T}_{k \mid k-1, m} . \\
0 & \text { else }\end{cases}
\end{aligned}
$$

Gaussian components are statistically closed to the tracks in the sense of the Mahalanobis distance.

Concerning the track deletion/birth process, two parameters have to be taken into account. First, the estimated number of targets $\hat{n}_{k}$ can change, second, a track can become improbable or the confidence in a Gaussian component can become very high. Consequently, the following rules for building the matrix $\tilde{A}_{k}$ are observed.

1) If a previous track cannot be associated with any Gaussian component at the current iteration, the track is deleted.

2) If the estimated number of targets decreases, the track which has the smallest score is deleted. (A definition of the track score can be found in [8]).

3 ) If the estimated number of targets increases or a previous track is deleted, a new track must be initialized. Each Gaussian component is also considered as a potential new track.

4) If a strongly weighted Gaussian component is not associated with a track, a previous track is deleted and a new track is initialized with this Gaussian component.
Knowing that a track is at most associated with one Gaussian component, the best association matrix $\mathbf{A}_{k}$ meets the following criteria:

$$
\left\{\begin{array}{l}
\sum_{m=1}^{\hat{N}_{k}^{\star}} \sum_{n=1}^{N_{k}^{\mathcal{G}}} \mathbf{A}_{k}(m, n)=\hat{n}_{k \mid k} \\
\forall n \leq N_{k}^{\mathcal{G}}, \quad \sum_{m=1}^{\hat{N}_{k}^{\star}} \mathbf{A}_{k}(m, n) \leq 1 . \\
\forall m \leq \hat{n}_{k}^{\star}, \quad \sum_{n=1}^{N_{k}^{\mathcal{G}}} \mathbf{A}_{k}(m, n)=1
\end{array}\right.
$$

The goal is to select this matrix $\mathbf{A}_{k}$ among the set $A_{k}=\left\{A_{k, a}\right\}_{a=1}^{N_{a}}$ of $N_{a}$ feasible associations at iteration $k$. Two criteria for selecting this matrix have been defined.

1) The more strongly weighted a Gaussian, the more plausible its correspondence is to a real target. On this assumption we want to select the set of Gaussian components which is as strongly weighted as possible and which produces the most plausible association of tracks.

2) The second criterion is also the cost of an association track/Gaussian component. The goal is to minimize the cost between a predicted track and a Gaussian component.

The weight matrix is defined as

$$
\forall n \leq N_{k}^{\mathcal{G}}, \quad W_{k}(:, n)=w_{k, i}^{\mathcal{G}}
$$

where $W_{k}(:, n)$ corresponds to the $n$th row of the matrix $W_{k}$ and $w_{k, i}^{\mathcal{G}}$ is the weight of the Gaussian component $\mathcal{G}_{k, i}$ (in other words, the peak intensity).

Finally, the global weight $W_{k, a}^{\mathcal{G}}$ of an association $a$ can be computed as the sum of the weights of the Gaussian components implied by the association tracks/Gaussian:

$$
W_{k, a}^{\mathcal{G}}=\sum_{m=1}^{\hat{N}_{k}^{\star}} \sum_{n=1}^{N_{k}^{\mathcal{G}}} A_{k, a}(m, n) \cdot W_{k}(m, n) .
$$

However, when targets are close together, the association which maximizes the global weight $W_{k, a}^{\mathcal{G}}$ may not be unique, and finally, the set of association matrices which maximize the global weight are written as

$$
A_{k}^{\star}=\underset{A_{k, a}}{\arg \max } W_{k, a}^{\mathcal{G}} .
$$

If $A_{k}^{\star}$ is unique, then $\mathbf{A}_{k}=A_{k}^{\star}$. If not, the cost of association track/Gaussian, based on the distance between predicted tracks and Gaussian means, is used in order to select one association matrix among $A_{k}^{\star}$.

In a similar way to the weight matrix $W_{k}$, the cost matrix $C_{k}$ can be calculated as $\forall m \in\left\{1, \ldots, N_{k}^{\mathcal{G}}\right\}$, $\forall n \in\left\{1, \ldots, \hat{N}_{k}^{\star}\right\}:$

$$
C_{k}(m, n)=c(m, n)
$$


where $c(m, n)$ is the cost of the association of the $m$ th track and the $n$th Gaussian component, written as the negative natural logarithm of the likelihood ratio:

$$
c(m, n)=-\ln \left(\frac{P_{\mathrm{d}} \cdot g\left(\mathcal{G}_{k, n} \mid \hat{x}_{k \mid k-1, j}\right)}{\beta_{\mathrm{fa}}}\right)
$$

where $g\left(\mathcal{G}_{k, n} \mid \hat{x}_{k \mid k-1, j}\right)$ is the likelihood of the Gaussian component $n$ given the predicted position $\hat{x}_{k \mid k-1, j}$ of the track $j$.

The global cost $C_{k, a}^{\mathcal{G}}$ of an association $a$ at each iteration $k$ is calculated as the sum of costs implied into an association matrix $a$ :

$$
C_{k, a}^{\mathcal{G}}=\sum_{m=1}^{\hat{N}_{k}^{\star}} \sum_{n=1}^{N_{k}^{\mathcal{G}}} A_{k, a}(m, n) \cdot C_{k}(m, n) .
$$

Finally, the best association $\mathbf{A}_{\mathbf{k}}$ is computed like the minimal cost matrix:

$$
\mathbf{A}_{\mathbf{k}}=\underset{A_{k}^{\star}}{\arg \min } C_{k, a}^{\mathcal{G}} .
$$

An optimization algorithm is also proposed in [3] for limiting the time complexity.

\section{Hybridization of CPHD filter with MHT technique with road constraints}

The labeled GM-CPHD filter presented in Section II-C is a powerful algorithm. Nevertheless, the state estimation precision cannot be better than the precision provided by a Kalman filter, and performances are worse concerning the velocity estimation because of the merging step used for the Gaussian component management [20]. In this section we propose an original answer to this problem. The labeled GM-CPHD filter is used as a track detector (Algorithm 1), whereas a second algorithm like MHT (Algorithm 2) is used to improve the state estimation, as shown in Fig. 3. It is worth noting that this algorithm should use the largest number of data sources possible. Moreover, by considering the scheme presented in Section II-C, it is conceivable to parallelize calculations for both algorithms. Thereby, Algorithm 2 combines a multi-model approach [21] using road map data provided by the GIS. The proposed algorithm, called the VS-IMMC-MHT (variable structure-interacting multiple model with constraints-multiple hypothesis tracker) [22], is an interacting multiple model (IMM) filter adapted to ground maneuvers, constrained to the road network and integrated in a structured branching-MHT (SB-MHT) [23]. In the next paragraph a short description of the variable structure-interacting multiple model under constraint (VS-IMMC) is given.

Our IMM is based on models constrained to the road segments. On the assumption that a vehicle is most likely moving on the road, estimated states are projected on the road segments, and velocity

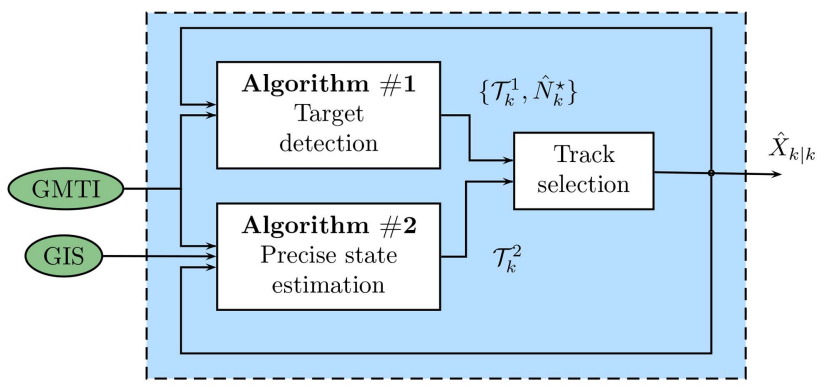

(a)

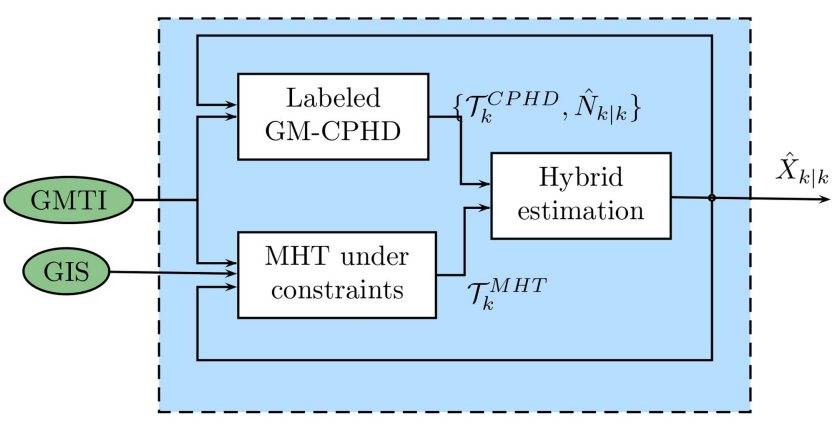

(b)

Fig. 3. Hybridization algorithm. (a) Principle scheme. (b) Implemented scheme.

vectors are constrained to the road segment direction, as well as their associated covariance matrices. In this way, during the prediction step, for each model, velocity vectors are successively constrained to the road segment direction, until the predicted distance is covered. Predicted covariance matrices are defined in the manner so that the standard deviation in the road segment direction is higher than the standard deviation in the orthogonal direction. In this application a three-model IMM is used to handle targets' move-stop-move strategies [24]: a constant velocity $(\mathrm{CV})$ model with low process noise standard deviation to deal with nonmaneuvring targets, another $\mathrm{CV}$ model with high process noise standard deviation to deal with maneuvring targets, and a stop model to deal with stopped targets.

If the predicted states are on several roads (in the case of a road intersection), several constrained motion model sets, called on-road models, are activated for each road intersection. The structure of the IMM is thus variable because it is adapted to the road network topology. In addition all the motion models of the IMM are constrained to the road. This is why the algorithm is named VS-IMMC.

After the IMM estimation step [25], estimated states of each motion model are projected on the most likely road segment and combined to provide the final estimated states.

However, if the target leaves the road, a classical off-road model can be used. A simple statistical test is then used to activate the off-road motion model set if the target is leaving the road network. 
Therefore, VS-IMMC contains a set of off-road models and several sets of on-road motion models in case of intersections. In order to limit the number of motion model sets, a sequential probability ratio test (SPRT) is used to confirm or delete a motion model set. If the test is not relevant to validate only one motion model set, the surviving sets are maintained, and the test is differed for the next scan.

The VS-IMMC is finally integrated in an SB-MHT [23].

The hybridization principle can finally be summarized as follows: the two algorithms, MHT and GM-CPHD, are used as complementary filters. The first estimates the number of targets and initializes the target positions. The second increases the accuracy for the target state estimation. The two algorithms are running simultaneously. Then, a gating process is applied around the target position given by the GM-CPHD filter to select MHT tracks. Finally, MHT tracks which have the highest scores are selected. If a CPHD track is not associated within any MHT track, the GM-CPHD track is kept. This approach combines the following advantages of the different algorithms without increasing the processing time:

1) robust to numerous ground target maneuvers by using a multi-model approach,

2) good precision for state estimation by using road coordinates,

3) management of road intersection,

4) precise estimation of the number of targets by using Cardinalized generalization of the PHD filter,

5) management of birth and death processes by using the new labeled implementation of the GM-CPHD filter.

All these advantages lead to a powerful algorithm. The main advantage is that no decrease in performance is observed when targets are close together (see Section IV).

\section{OBJECT-OF-INTEREST DETECTION}

In the context of battlefield surveillance, some strategic objects of interest are observed to assess the situation. A particular type of object is a convoy which is particularly difficult to detect because it is composed of vehicles seen as an aggregate with a particular behavior. Properties describing a convoy are mainly based on the positions and kinematics of the vehicles which compose the aggregate: a convoy is defined as a vehicle set evolving approximately with the same dynamics over a long period of time. These vehicles are moving on the road at a limited velocity $(<20 \mathrm{~m} / \mathrm{s})$. They must stay within sight of each other with almost constant distances between them (mostly $100 \mathrm{~m}$ ). Moreover, these criteria must be added to context and type properties.

Vehicle positions and kinematics are computed by the algorithm presented in Section II. From the set
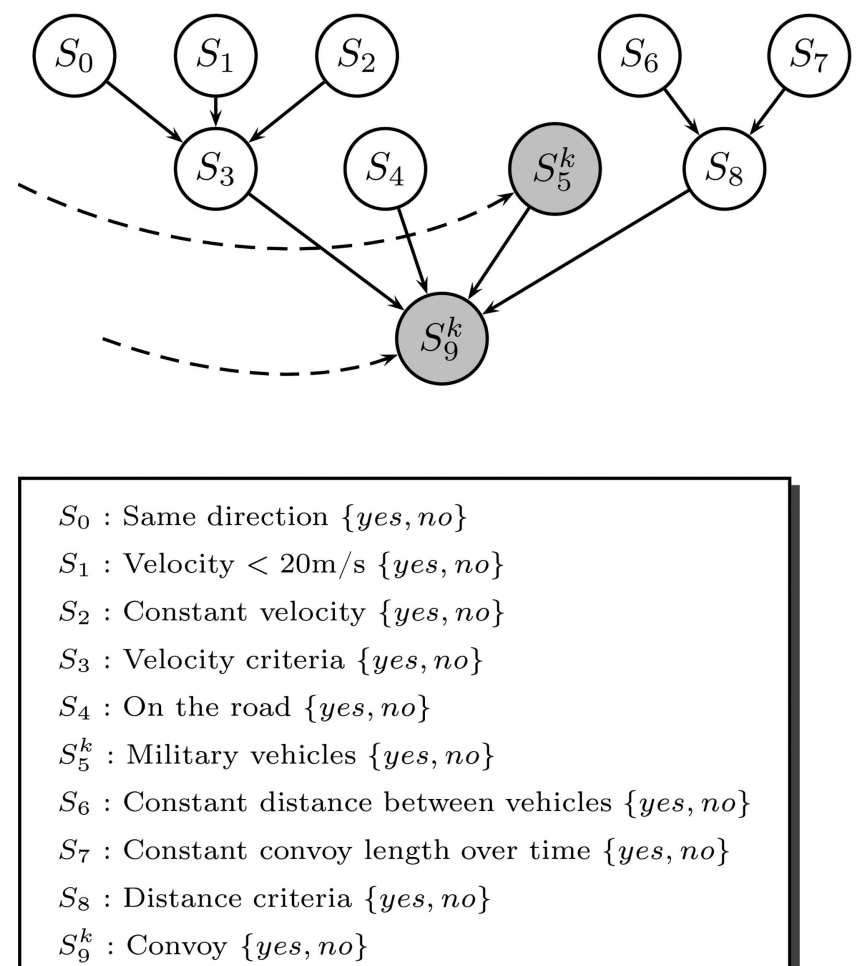

Fig. 4. Convoy model. Grey nodes are time dependent.

of detected vehicles, we first detect the aggregates $\mathcal{A}_{k}$ at time $k$ as groups of closely spaced targets, and each aggregate is considered a convoy if it satisfies all the criteria. That is why it is essential to use outputs coming from a precise MTT algorithm.

The criteria are manifold and of different natures. Data are heterogeneous, asynchronous, and can be missing. Moreover, random variables are continuous (state), discrete (type), and time dependent. The graphical models represent an interesting formalism in object-of-interest detection (OID) and have already been used in similar topics [26-29]. Graphical models are traditionally used to represent dependency relations between a set of $N$ random variables. Graphically, each variable is represented by a node, and an arc from a node $S_{i}$ to a node $S_{j}$ means that $S_{i}$ "causes" $S_{j}, \forall(i, j) \in\{1, \ldots, N\}^{2}$. According to the convoy definition, the object-of-interest convoy is modeled by nine nodes including velocity, positioning, type, or contextual criteria (Fig. 4).

The main usual graphical model is the Bayesian network. In this model each node $i$ is associated with a probability function $P\left(S_{i}\right)$ that gives the probability of the variable $S_{i}$ represented by the node. At each iteration it is possible to compute the evolution of the probabilities of the graph by taking into account all updated inputs. Finally, the joint probability at iteration $k$ is computed as

$$
\mathrm{P}\left\{S_{1}, \ldots, S_{9}\right\}=\sum_{i=1}^{9} \mathrm{P}\left\{S_{i} \mid \operatorname{Pa}\left(S_{i}\right)\right\}
$$

where $\operatorname{Pa}\left(S_{i}\right)$ represents the parent nodes of $S_{i}$. 
The goal is to calculate at each GMTI iteration the probability $\mathrm{P}\left\{S_{9}^{k}\right\}$ for a detected aggregate to be a convoy (this node is in gray in Fig. 4). Moreover, we want to take into account the time evolution of random variables. And, concerning the node $S_{9}^{k}$, the convoy presence is confirmed with time. The variable $S_{5}^{k}$ is time dependent too because the type information can come from heterogeneous sources $(\mathrm{SAR}$, video, ...) with different scanning times.

\section{A. Recall of Credibilistic Framework}

The transferable belief model (TBM) developed by P. Smets [30] and T. Denoeux [31] is an alternative to the probabilistic approach. It is a well-adapted framework to model knowledge about a complex system. In this framework the main idea is to assign a belief distribution $m($.) on a variable to a larger set as with probabilities, denoted the power set. For the OID we developed a credibilistic network where each node $S_{i}$ is associated with a belief distribution [32].

We consider a state space $\Omega_{i}=\left\{S_{i}, \overline{S_{i}}\right\}$ where $\overline{S_{i}}$ means that the state $S_{i}$ is not true. The power set $2^{\Omega_{i}}=\left\{\varnothing,\left\{S_{i}\right\},\left\{\overline{S_{i}}\right\}, \Omega_{i}\right\}$, of size $2^{\left|\Omega_{i}\right|}$, is jointly composed of hypotheses and joined hypotheses $\Omega_{i}$. Finally, a basic belief assignment $m^{\Omega}(B)$, also named $\mathrm{BBA}$, is computed for each element of the power set $2^{\Omega}$, according to the following equation:

$$
\sum_{B \in 2^{\Omega}} m^{\Omega}(B)=1
$$

In this framework other belief functions are defined on the power set such as the plausibility function $p l^{\Omega}(B)$ ([30]) or the communality $q$ which are elementary functions in one-to-one correspondence with the belief mass $m^{\Omega}(B)$ defined in (21). All these functions have a conditional form such as $m^{\Omega}[A](B)$ meaning the confidence about $B$ given that $A$ is true.

1) Combination Rules: One important aspect for using the TBM framework in graphical models lies in the way that information from the different nodes seen as different sources of information is combined. We bring to mind in (22) and (23) two classical combination rules which are widely used in this section. The first is called the conjunctive rule of combination (CRC). It is an associative and commutative operation that combines belief distributions $m_{1}^{\Omega}$ and $m_{2}^{\Omega}$ coming from reliable and independent sources:

$$
m_{1 @ 2}^{\Omega}(A)=\left(m_{1}^{\Omega} @ m_{2}^{\Omega}\right)(A)=\sum_{B \cap C=A} m_{1}^{\Omega}(B) \cdot m_{2}^{\Omega}(C)
$$

where $A, B, C \subset \Omega$.

The second one is called the disjunctive rule of combination (DRC). It is defined as the combination rule for unreliable sources or a rule able to deal with conflict:

$$
m_{1(1)}^{\Omega}(A)=\left(m_{1}^{\Omega}(\mathrm{S}) m_{2}^{\Omega}\right)(A)=\sum_{B \cup C=A} m_{1}^{\Omega}(B) \cdot m_{2}^{\Omega}(C) .
$$

2) Temporal Belief Filter: As for dynamic

Bayesian networks, the temporal aspect must be specifically handled. To ensure the temporal consistency, a temporal belief filter, first defined by Ramasso [33], is then applied. Assuming that each node $S_{i}$ is a binary node $\left(\Omega_{i}=\left\{S_{i}, \bar{S}_{i}\right\}\right)$, the following vector notation for the belief distribution $m^{\Omega_{i}}$ is used

$$
m^{\Omega_{i}}=\left[\begin{array}{llll}
m^{\Omega_{i}}(\varnothing) & m^{\Omega_{i}}\left(S_{i}\right) & m^{\Omega_{i}}\left(\bar{S}_{i}\right) & m^{\Omega_{i}}\left(\Omega_{i}\right)
\end{array}\right]^{T} .
$$

On the assumption that a temporal node can be viewed as a nonstationary system, whose temporal evolution can be modeled according to an evolution matrix $F_{k}^{\Omega_{i}}$, the predicted BBA $\hat{m}_{k \mid k-1}^{\Omega_{i}}$ on that node $S_{i}$ at time $k$ can be written as

$$
\hat{m}_{k \mid k-1}^{\Omega_{i}}=F_{k}^{\Omega_{i}} \cdot \hat{m}_{k-1 \mid k-1}^{\Omega_{i}}
$$

where $\hat{m}_{k-1 \mid k-1}^{\Omega_{i}}$ is the estimated BBA on node $S_{i}$ at previous time $k-1$.

The temporal evolution matrix $F^{\Omega_{i}}$ of size $2^{\left|\Omega_{i}\right|} \times$ $2^{\left|\Omega_{i}\right|}$ is written as

$$
F^{\Omega_{i}}=\left[\begin{array}{llll}
F^{\Omega_{i}}(\varnothing) & F^{\Omega_{i}}\left(X_{i}\right) & F^{\Omega_{i}}\left(\bar{X}_{i}\right) & F^{\Omega_{i}}\left(\Omega_{i}\right)
\end{array}\right]
$$

where $F^{\Omega_{i}}(\varnothing)=\left[\begin{array}{llll}1 & 0 & 0 & 0\end{array}\right]^{T}$ and $F^{\Omega_{i}}\left(\Omega_{i}\right)=\left[\begin{array}{llll}0 & 0 & 0 & 1\end{array}\right]^{T}$ because all conflict/doubt is transferred on itself. The term $F^{\Omega_{i}}\left(S_{i}\right)$ (resp. $\left.F^{\Omega_{i}}\left(\bar{S}_{i}\right)\right)$ represents the model evolution of the node $S_{i}$ if its value is true (resp. false). In this case the belief on $S_{i}$ (resp. $\bar{S}_{i}$ ) is partly transferred on $S_{i}$ (resp. $\bar{S}_{i}$ ) according to a certain confidence $\alpha_{\mathcal{T}}$ (resp. $\alpha_{\mathcal{F}}$ ) and the rest on the doubt $\Omega_{i}$ as

$$
\begin{aligned}
& F^{\Omega_{i}}\left(S_{i}\right)=\left[\begin{array}{llll}
0 & \alpha_{\mathcal{T}} & 0 & 1-\alpha_{\mathcal{T}}
\end{array}\right]^{T} \\
& F^{\Omega_{i}}\left(\bar{S}_{i}\right)=\left[\begin{array}{llll}
0 & 0 & \alpha_{\mathcal{F}} & 1-\alpha_{\mathcal{F}}
\end{array}\right]^{T} .
\end{aligned}
$$

Finally, the obtained predicted belief distribution is combined with the measured belief distribution $\tilde{m}_{k}^{\Omega_{i}}$ coming from data at time $k$. This combination is made according to a CRC (cf. (22)) because predicted and estimated belief distributions are independently calculated. This highlights the conflict between prediction and measurement and is written as

$$
\hat{m}_{k \mid k}^{\Omega_{i}}=\tilde{m}_{k}^{\Omega_{i}} @ \hat{m}_{k \mid k-1}^{\Omega_{i}} .
$$

\section{B. Dynamic Evidential Network Inference}

We have developed an evidential inference mechanism in order to deal with graphical models such as Bayesian networks. This framework is very suitable for this application. The two initialization steps are first described. 
1) Prior Mass Belief Establishment: With the Bayesian approach the first step would be to establish $p\left(S_{i} \mid P a\left(S_{i}\right)\right)$ of size $2 \times\left|P a\left(S_{i}\right)\right|$ where $P a\left(S_{i}\right)$ is a parent node of node $S_{i}$ but whose size can quickly increase depending on the number of feasible states for parent nodes and more generally on the number of parent nodes. With evidential networks the conditional beliefs $m^{\Omega_{i}}\left[S_{i}\right]$ are established for each parent node $j$ and independently of the others according to the knowledge on the system. However, only conditional belief functions on $S_{i}$ knowing that $S_{j}$ is in the state $S_{i}$ or $\bar{S}_{i}$ can be established. The belief that the node $S_{j}$ is in the state $\Omega_{j}$ cannot be intuitively established but is computed by using the DRC as in (23).

2) Discounting Coefficient Establishment: When a node depends on many other nodes, it is possible to decrease the importance of a node in comparison with another node by using discounting coefficients [34]. Another point of view could be that the parent nodes can be seen as independent sources which are strongly or weakly weighted, depending on their reliability.

When the evidential network is described, the inference mechanism can be used. It is now decomposed in five elementary operations.

1) Data Transformation: For probabilistic networks this critical operation is very seldom described in the literature. The belief in one hypothesis is calculated for each root node by using fuzzy sets or statistical distributions, like the Rayleigh distribution, as described in [35]. We propose to establish the belief distribution according to

$$
\left\{\begin{array}{l}
\tilde{m}^{\Omega_{i}}(\phi)=0 \\
\tilde{m}^{\Omega_{i}}\left(S_{i}\right)= \begin{cases}\mathrm{P}\left\{S_{i}\right\} & \text { if } \quad \mathrm{P}\left\{S_{i}\right\} \geq 0.5 \\
0 & \text { else }\end{cases} \\
\tilde{m}^{\Omega_{i}}\left(\bar{S}_{i}\right)= \begin{cases}\mathrm{P}\left\{\bar{S}_{i}\right\} & \text { if } \mathrm{P}\left\{\bar{S}_{i}\right\}>0.5 \\
0 & \text { else }\end{cases} \\
\tilde{m}^{\Omega_{i}}\left(\Omega_{i}\right)= \begin{cases}1-\mathrm{P}\left\{S_{i}\right\} & \text { if } \quad \mathrm{P}\left\{S_{i}\right\} \geq 0.5 \\
1-\mathrm{P}\left\{\bar{S}_{i}\right\} & \text { else }\end{cases}
\end{array}\right.
$$

2) Propagation: The BBAs coming from each parent node $S_{i}$ are propagated to child nodes $S_{j}$. The propagated BBA from node $i$ to $j$ is written as $m_{i \rightarrow j}^{\Omega_{j}}$. It is calculated by using conditional plausibility $p l^{\Omega_{j}}[C](B)$ where $C \subseteq \Omega_{i}$ and $B \subseteq \Omega_{j}$ [30], which is more convenient for the use of the generalized Bayes theorem (GBT) written as

$$
p l_{i \rightarrow j}^{\Omega_{j}}(B)=\sum_{C \subseteq \Omega_{j}} p l^{\Omega_{j}}[C](B) \cdot \tilde{m}^{\Omega_{i}}(C) .
$$

3) Discounting: If discounting coefficients are filled in as presented in the second initialization step, the discounting formula is applied on propagated belief distributions $m_{i \rightarrow j}^{\Omega_{j}}$ to obtain ${ }^{\alpha_{i}} m_{i \rightarrow j}^{\Omega_{j}}$. This $\alpha$ discounting process is generally defined by

$$
\begin{aligned}
{ }^{\alpha} m^{\Omega}(A) & =(1-\alpha) m^{\Omega}(A) \quad \forall A \subset \Omega \\
{ }^{\alpha} m^{\Omega}(\Omega) & =(1-\alpha) m^{\Omega}(\Omega)+\alpha
\end{aligned}
$$

if $\Omega$ is a binary set.

4) Combination: Discounted propagated belief distributions from parent nodes $i$ and $j$ are finally combined by using the CRC with communality $q^{\Omega_{j}}$, which is more convenient for the calculation of the $\mathrm{CRC}$ as in (22):

$$
\tilde{q}^{\Omega_{j}}\left(S_{j}\right)={ }^{\alpha_{i}} q_{i \rightarrow j}^{\Omega_{j}}\left(S_{j}\right) \cdot{ }^{\alpha_{l}} q_{l \rightarrow j}^{\Omega_{j}}\left(S_{j}\right) .
$$

It must be noticed that the predicted belief (25) for nodes 5 and 9 are considered at this step as parent nodes.

5) Estimation: Finally, the measured commonality distribution $\tilde{q}^{\Omega_{j}}($.$) is converted into a belief mass$ distribution and used to update the BBA of node $S_{9}$ according to (28).

\section{Number of Targets of the Convoy}

As for the targets it is necessary to track convoys. The specificity is that the convoy structure can change over time, especially the number of targets belonging to the convoy.

Let $\mathcal{A}_{k}$ define a detected aggregate at iteration $k$ characterized by its barycenter $\hat{x}_{k}^{\mathcal{A}}$, its covariance $P_{k}^{\mathcal{A}}$, and its number of targets $N^{\mathcal{A}_{k}}$. The probability of having a convoy is denoted $\mathrm{P}\left\{S_{9}^{k} \mid N^{\mathcal{A}_{k}}\right\}$.

At iteration $k+1$, another aggregate $\mathcal{A}^{\prime}$ (of barycenter $\hat{x}^{\prime}$, covariance $P^{\prime}$, and cardinality $N^{\mathcal{A}_{k+1}}$ ) is detected. The idea is now to check if $\mathcal{A}^{\prime}$ is in correspondence with $\mathcal{A}_{k}$. The predicted position and covariance, denoted $\hat{x}_{k+1 \mid k}^{\mathcal{A}}$ and $P_{k+1 \mid k}^{\mathcal{A}}$, are then calculated according to (33) and (34) at iteration $k+1$ :

$$
\begin{gathered}
\hat{x}_{k+1 \mid k}^{\mathcal{A}}=F^{\mathcal{A}} \cdot \hat{x}_{k}^{\mathcal{A}} \\
P_{k+1 \mid k}^{\mathcal{A}}=F^{\mathcal{A}} \cdot P_{k}^{\mathcal{A}} \cdot\left(F^{\mathcal{A}}\right)^{T}+Q^{\mathcal{A}}
\end{gathered}
$$

where $F^{\mathcal{A}}$ is the convoy transition matrix similar to the target transition model defined in (3) and $Q^{\mathcal{A}}$ defines the model uncertainty as in (4).

A gating process is then used. If the two aggregates $\mathcal{A}_{k}$ and $\mathcal{A}^{\prime}$ satisfy the following condition, then they are in correspondence:

$$
\left(\hat{x}_{k+1 \mid k}^{\mathcal{A}}-\hat{x}^{\prime}\right)^{T}\left(P_{k \mid k-1, j}^{\mathcal{A}}\right)^{-1}\left(\hat{x}_{k+1 \mid k}^{\mathcal{A}}-\hat{x}^{\prime}\right)<\gamma
$$

with the probability threshold $\gamma$, obtained from the chi-square tables.

They form an aggregate sequence denoted $\mathcal{A}_{k: k+1}$ of cardinality $N_{k: k+1}^{\mathcal{A}}$. The probability of having a convoy can be calculated as $\mathrm{P}\left\{S_{9}^{k} \mid N^{\mathcal{A}_{k}}\right\}$. It is now easy to understand that the different numbers of targets considered in the convoy in 
the set of number $N_{k: k+1}^{\mathcal{A}}$ can be seen as different hypotheses for the number of targets belonging to the convoy. The probability of having a convoy is also jointly calculated for the different number of target hypotheses and is noted $\mathrm{P}\left\{S_{9}^{k} \mid N_{k_{0}: k}^{\mathcal{A}}, N_{i}^{\mathcal{C}}\right\}$, with $N_{i}^{\mathcal{C}}$ belonging to the set of number of target hypotheses.

\section{SIMULATION RESULTS}

In order to evaluate MTT algorithm performances, a high-quality GMTI sensor simulator was developed at Onera, and a realistic scenario was chosen containing several moving targets on a road map. The simulated measurements have very similar technical characteristics to the real ones (as shown in [36]). The typical measurement error is $20 \mathrm{~m}$ in range and 0.45 in azimuth. The false alarm density is $\beta_{\mathrm{fa}}=$ $8.92 \cdot 10^{-9}$ and the detection probability $P_{\mathrm{d}}$ is equal to 0.9 . The scanning time is $\Delta=10 \mathrm{~s}$. Experiments of various levels of difficulty have been carried out. The parameters of MTT and convoy detection algorithms are very well detailed in [37].

Performances are calculated for two critical situations. In the first scenario a slowing down of the traffic is observed due to the presence of a tractor creating an aggregate. In the second scenario an 8-target convoy separated into two 4-target convoys. For both scenarios MTT is first evaluated by using the well-known OSPA distance (optimal subpattern assignment) [38]. Convoy detection results are then analyzed, by considering the estimation of the number of targets in the convoy, with the probabilistic and the credibilistic graphical models.

\section{A. Scenario 1: Tractor Slowing Down}

1) Scenario Description: To analyze the performances of the algorithms described above, the following scenario representation is chosen (cf. Fig. 5). Fourteen targets, mainly cars except target 1 which is a tractor, are present in the observation zone. A single convoy, labeled $\mathrm{C} 1$, composed of jeeps (target 3 to 6 ) is to be detected. The scenario time is limited to $600 \mathrm{~s}$. In this scenario the target maneuvers are very elaborate, and the aggregate nature is complex to analyze. In fact the scenario contains one real convoy, but also a false one, arising from one of the vehicles slowing down. Key factors of the scenario are the following.

1) Targets 3-6 form convoy $\mathrm{C} 1$ moving on the main road from North to South.

2) Target 1 is a tractor, moving very slowly on the same road but in the opposite direction. It is passed by targets 2 and 10 and then slows down. Targets 7-9 are following the tractor but cannot overtake it as they are waiting for the approaching convoy to pass by.

3) Targets 11-13 are totally independent.

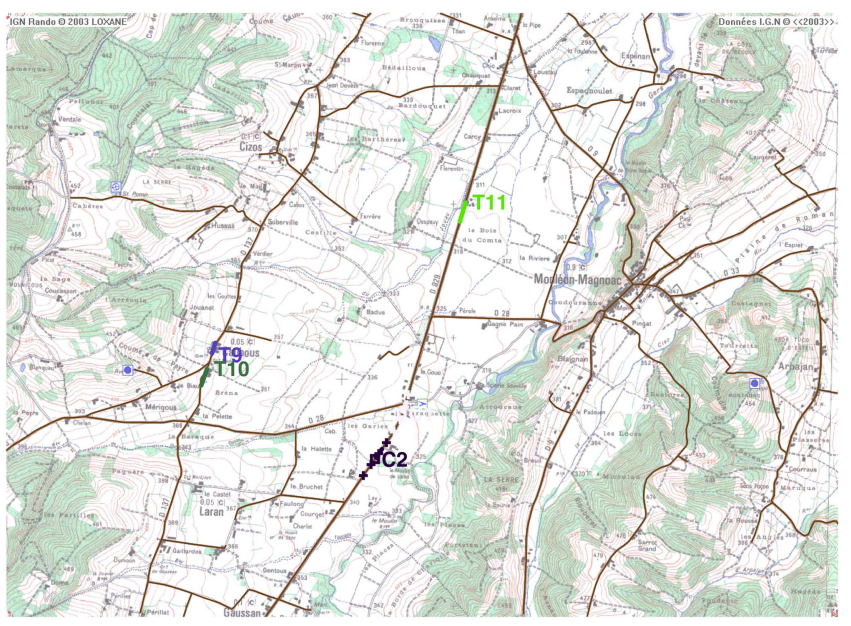

Fig. 5. Scenario 1 at time $t=101 \mathrm{~s}$. Targets 3-6, 10, 14 are moving from North to South on the main road. Targets 1, 2, 7-9 are moving from South to North on the main road. These targets are blocked by the tractor, they cannot pass it and consequently have the same behavior of a convoy. Finally, after passing the real convoy, they can pass the tractor on the right way. Other targets (T11-13) are moving independently on smaller roads.

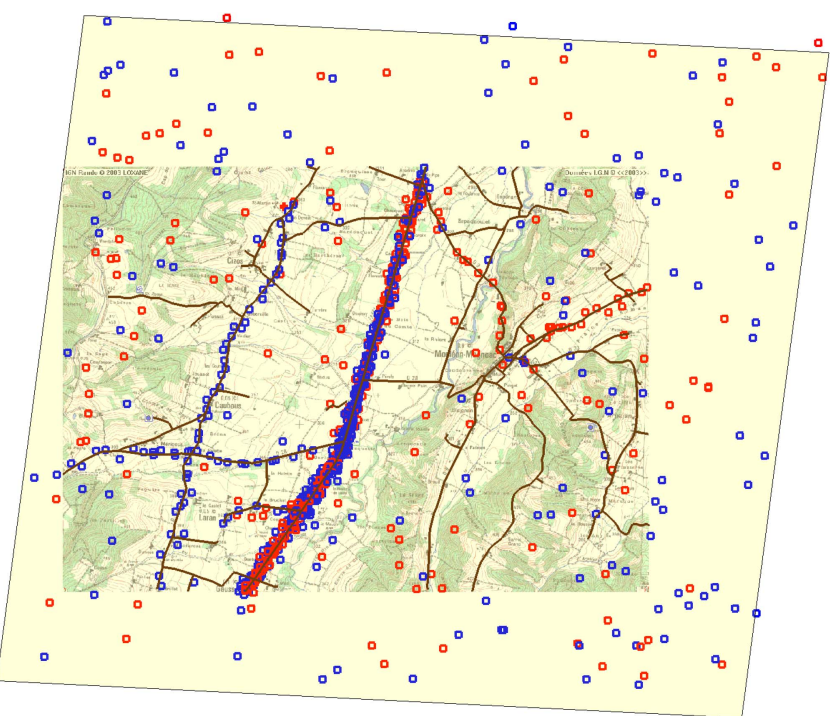

Fig. 6. Cumulated MTI reports: $\square$ MTI report with positive Doppler, $\square$ MTI report with negative Doppler, - Road, Observation zone.

4) Target 14 stops at an intersection in order to let the convoy pass by. No measurements are available between time $t=361 \mathrm{~s}$ and $t=481 \mathrm{~s}$.

For this scenario the GMTI sensor has a linear trajectory, and its altitude is $4000 \mathrm{~m}$. The cumulated MTI reports are shown in Fig. 6.

2) Tracking Performance Evaluation: Figure 7 presents results for the hybrid MTT algorithm (in pink) proposed in this paper, in comparison with an MHT with road constraints (in green) and a GM-CPHD with labeled tracks (in red). Results are averaged over 100 Monte-Carlo runs. The first subfigure presents results for cardinality estimation: at any time, the number of targets is evaluated in 

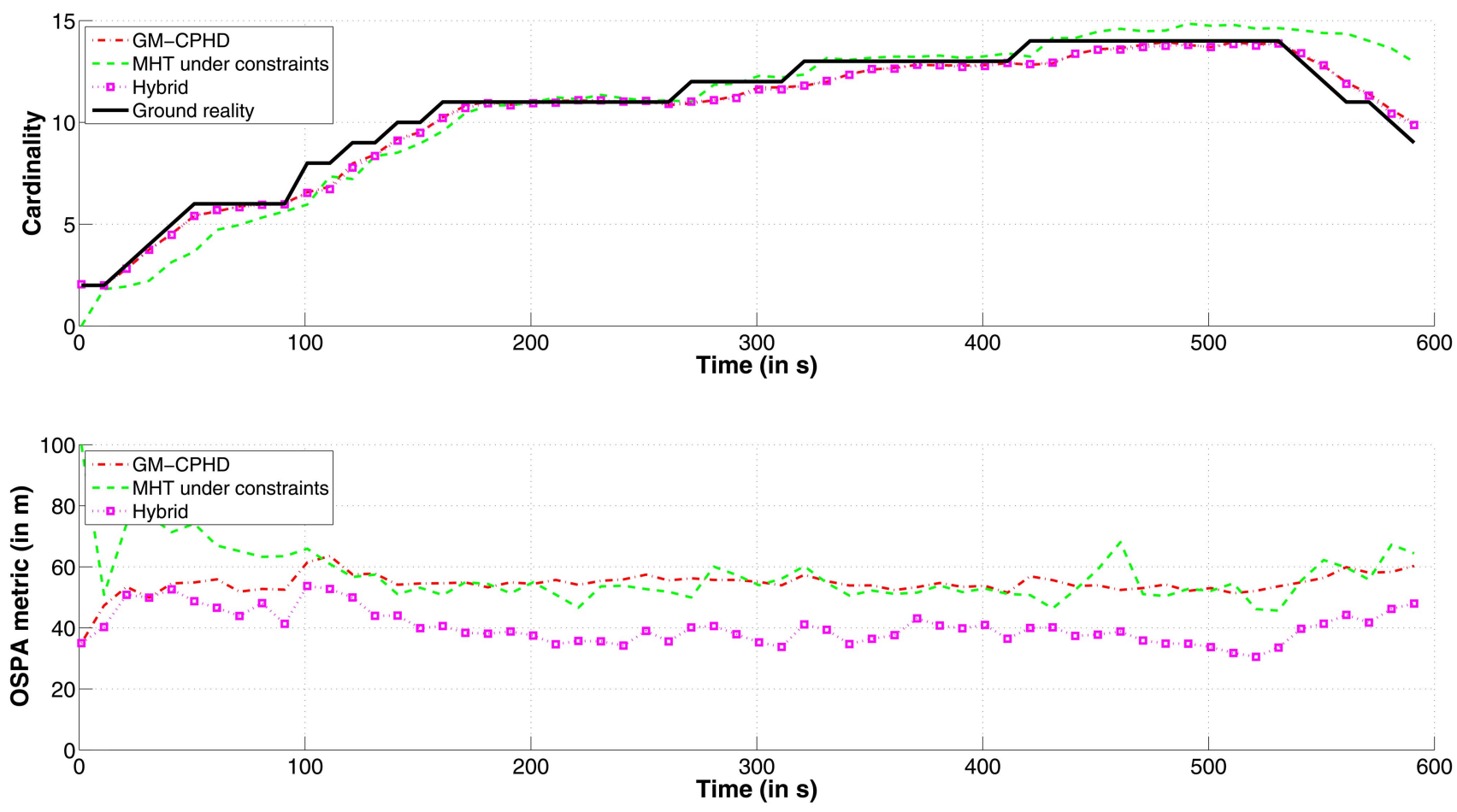

Fig. 7. MTT. Cardinality of detected targets and OSPA distance from ground truth.

comparison with the real number of targets created by the simulator. The hybrid algorithm and the GM-CPHD filter have a similar shape. They are both closer to the ground truth than the MHT with road constraints. They are more reactive to cardinality changes. Concerning the OSPA distance it is considerably lower for the hybrid algorithm than the MHT algorithm, and it is more stable during the cardinality changes (for example, between time $t=410$ and $t=430 \mathrm{~s}$ ). The OSPA distance for the labeled GM-CPHD filter is quite close to the MHT with road constraints, but they cannot be compared because the GM-CPHD does not use the road position as a priori.

3) Convoy Detection and Evaluation Results: The final objective of the algorithm is to detect the convoy and to estimate the number of targets $N$ belonging to it. We bring back to mind that $m(S)$ and $m(\Omega)$ represent the belief and the doubt that the aggregate is a convoy. The fusion process can generate a conflict value denoted as $m(\varnothing)$. In a probabilistic way $P(S)$ represents the probability that the aggregate is a convoy. During the simulation two aggregates are detected:

1) the aggregate corresponding to the real 4-target convoy $\mathrm{C} 1$,

2) the aggregate created by the tractor corresponding to a false alarm. Between time $t=250$ and $t=400 \mathrm{~s}$, targets 7-9 are blocked by the tractor; they cannot pass it and consequently have the same behavior as a convoy. Finally, after the real convoy has passed, they can overtake the tractor (between time $t=400$ and $t=570 \mathrm{~s}$ ).

Results for convoy detection and evaluation are summarized in Fig. 8. Figures 8(a) and (b) present credibilistic results compared with the probabilistic results (Figs. 8(c) and (d)). Figures 8(a) and (c) compare results obtained for the aggregate created by the tractor with results obtained for the real convoy (Figures 8(b) and (d)).

Concerning the aggregate with the tractor (false alarm), the probability of having a convoy is quite regular over time (Fig. 8(c)). With the credibilistic approach results are more contrasting (Fig. 8(a)). At the beginning of the aggregate detection (between time $t=180$ and $t=250 \mathrm{~s}$ ), the belief of having a convoy is quite low. Indeed, at this time, targets decelerate because of the tractor but are not blocked by it. Between time $t=250$ and $t=400 \mathrm{~s}$, the belief and the probability of having a convoy are high when the targets are blocked by the tractor. Finally, after passing by the real convoy, they can pass the tractor on the right side and the belief of having a convoy decreases (between time $t=400$ and $t=570 \mathrm{~s}$ ). This situation also appears by regarding the conflict (Fig. 8(e)). The conflict is quite high except between time $t=250$ and $t=400 \mathrm{~s}$, when the aggregate acts like a convoy.

Concerning the aggregate with the real convoy, from the beginning to the end, the convoy consisting of four targets is detected with high probability and belief. However, as for the tractor convoy, results are more contrasted with belief than with probabilities. 


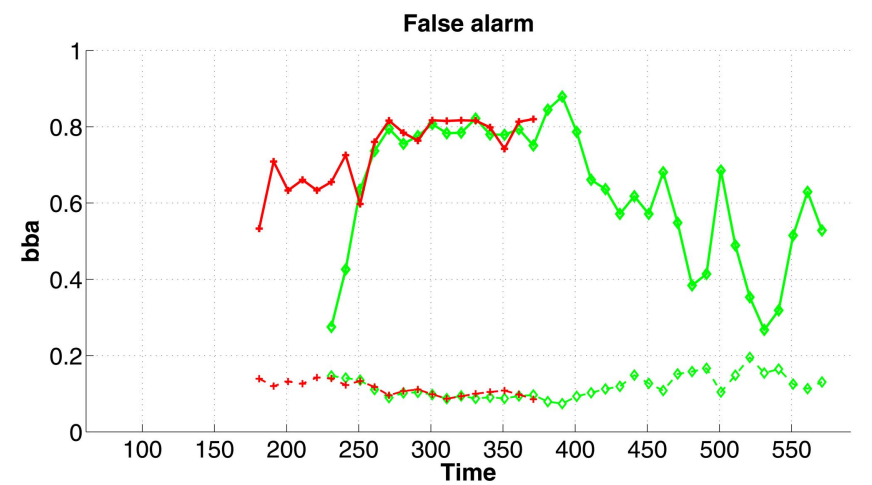

(a)

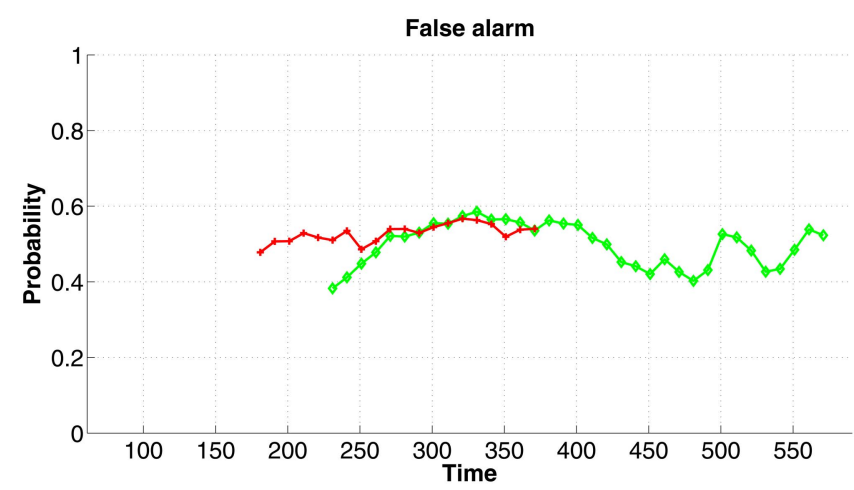

(c)

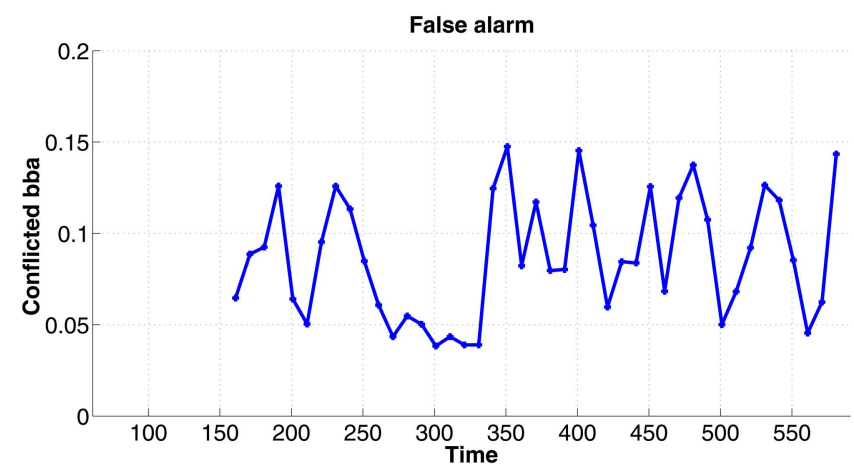

(e)

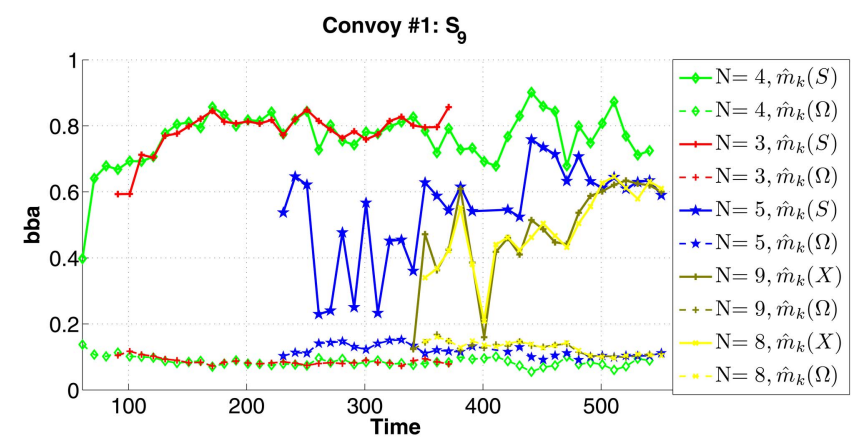

(b)

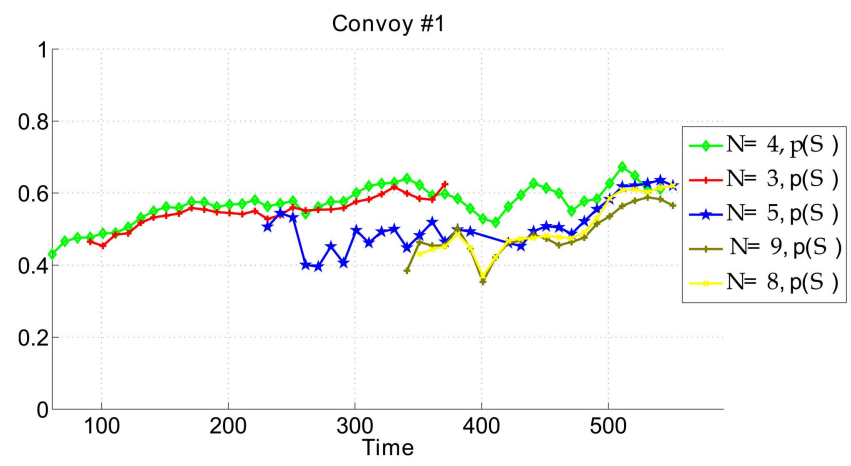

(d)

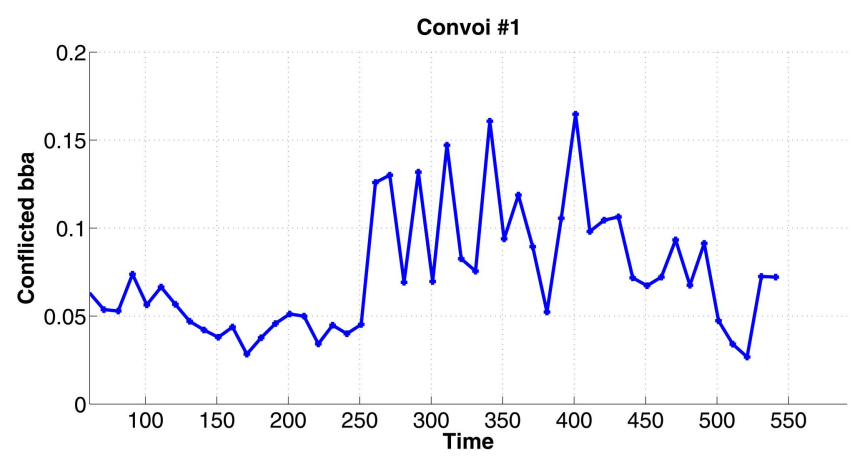

(f)

Fig. 8. Confidence and probabilities for different values of cardinality $N$ (number of targets in convoy). Conflict from fusion process: low when behavior corresponds to convoy. (a) Confidence on aggregate created by tractor. It corresponds to convoy of 4 targets between $t=270$ and $340 \mathrm{~s}$. (b) Confidence on real convoy of 4 targets. (c) Probabilities on aggregate created by tractor.

(d) Probabilities on real convoy of 4 targets. (e) Conflict detected from aggregate created by tractor. (f) Conflict detected from real convoy.

The belief of having a convoy consisting of three, four, five, eight, and nine targets is calculated. For the entire duration of the simulation, the belief of having a 4-target convoy is very high (higher than 0.8 ), however with probabilities, it has the same order of magnitude compared with the false alarm convoy. The number of targets in the convoy is therefore better discriminated with belief functions. Finally, the conflict is quite interesting for this case. It is quite low at the beginning of the simulation (between time $t=0$ and $t=250 \mathrm{~s}$ ) when the convoy evolves without any interaction with other vehicles. Doubt and conflict increase when targets are maneuvering (passing, overtaking, ...).
This scenario illustrates that the probabilistic approach is limited when the aggregates are a false alarm convoy (probability close to 0.5 ). The credibilistic approach is more reliable in characterizing doubt, identifying convoy maneuvers, and in distiguishing real convoys from false alarms.

\section{B. Scenario 2: Separating Convoy}

1) Scenario Description: Scenario 2 contains eleven targets, including an 8-target convoy which splits into two 4-target convoys. The scenario time is limited to 500s. Target trajectories are illustrated in Fig. 9, and the cumulated MTI reports are shown in Fig. 10. 


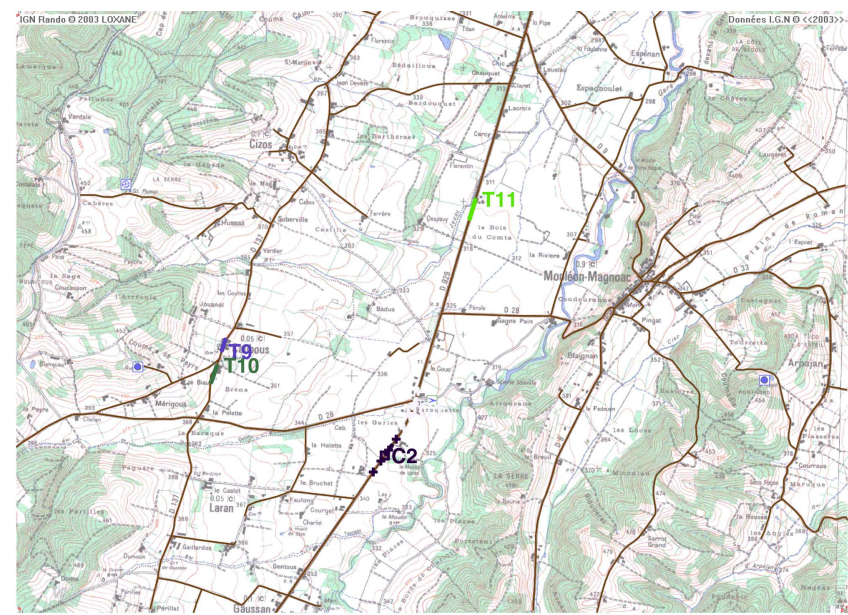

Fig. 9. Scenario 2: target trajectories.

Key actions during that scenario are the following.

1) Targets $1-8$ form a convoy from $t=1 \mathrm{~s}$ to $t=301 \mathrm{~s}$, moving on the main road from South to North.

2) Target 11 passes the convoy between time $t=241 \mathrm{~s}$ and $t=291 \mathrm{~s}$.

3) At time $t=301 \mathrm{~s}$, the convoy splits into two independent convoys; the last four targets turn on the right, while the four head targets continue on the main road. Special attention was paid to give realistic dynamics to the targets. They decelerate before turning, turn, and then accelerate again.

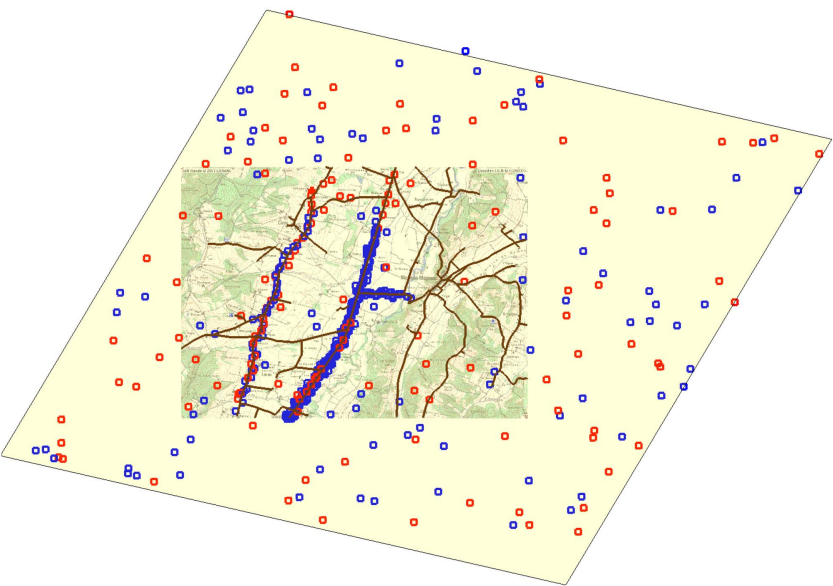

Fig. 10. Scenario 2: cumulated MTI reports - $\square$ MTI report with positive Doppler, $\square$ MTI report with negative Doppler, - Road, Observation zone.

4) Targets 9 and 10 are moving on another road than the convoy. They cross each other between time $t=161 \mathrm{~s}$ and $t=181 \mathrm{~s}$.

2) Tracking Performance Evaluation: Tracking results for scenario 2 are presented in Fig. 11. As in Fig. 7 results are compared for the hybrid MTT algorithm (in pink) proposed in this paper, for the MHT with road constraints (in green), and for the GM-CPHD with labeled tracks (in red). Results are very similar to the ones presented in scenario 1 . The hybrid algorithm and the GM-CPHD filter have a
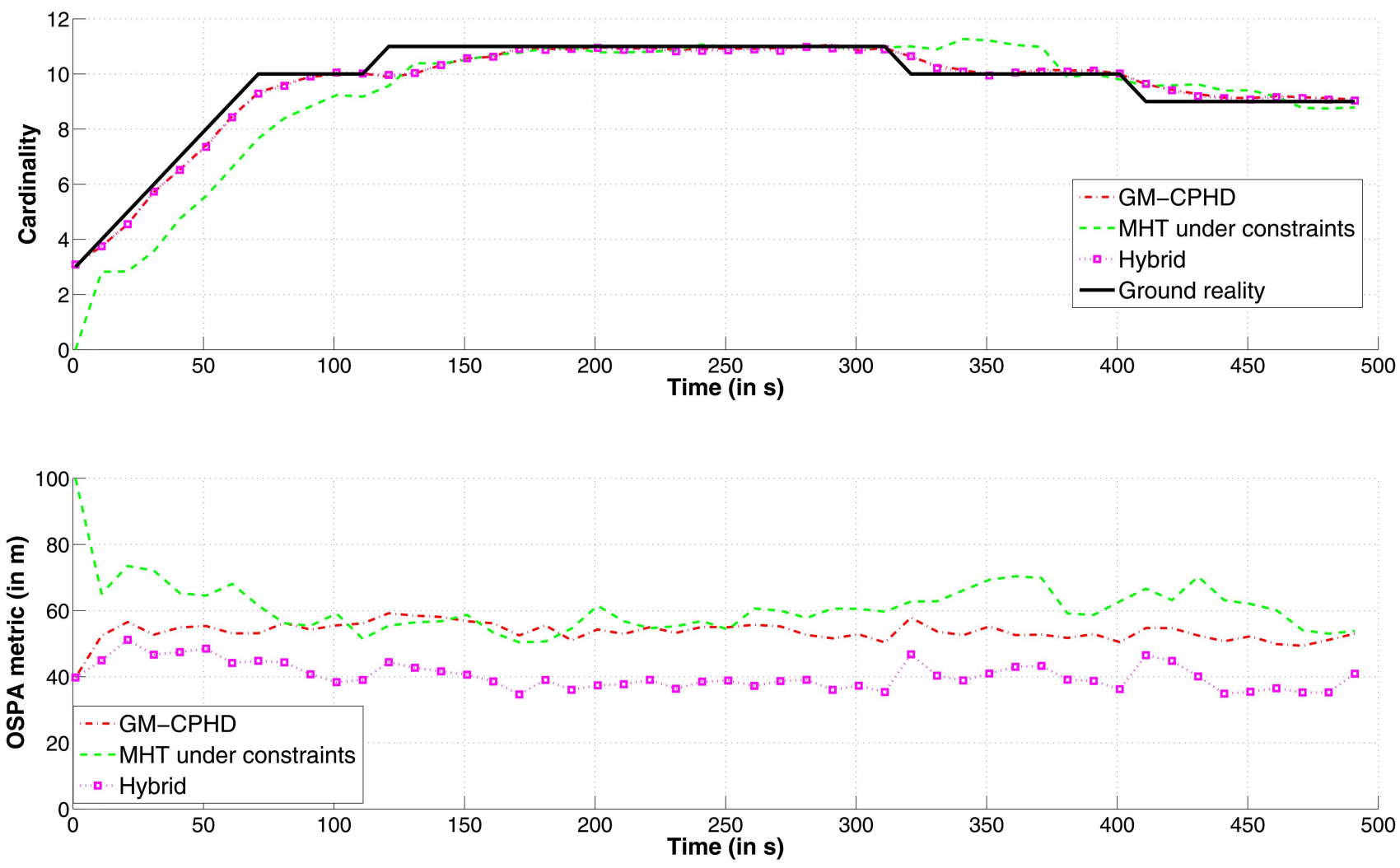

Fig. 11. MTT results. 


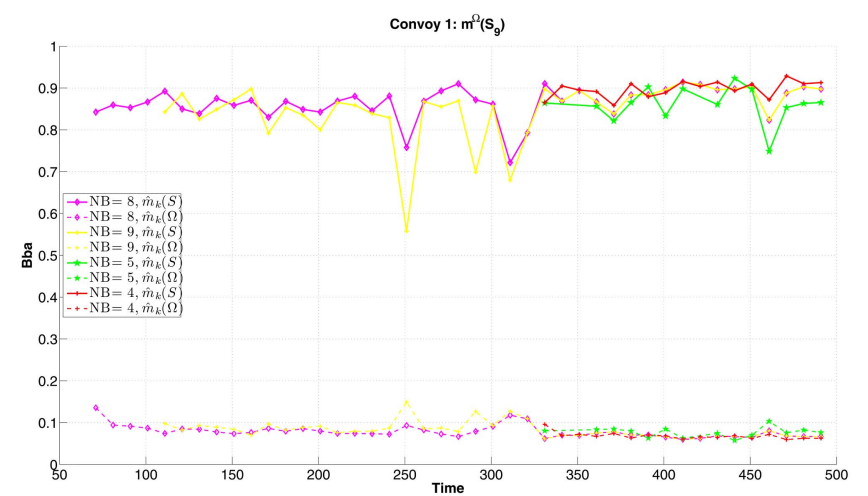

(a)

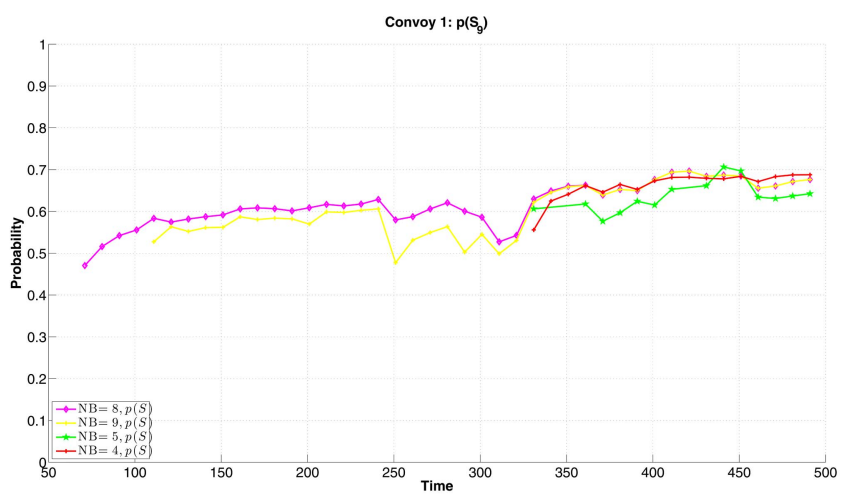

(c)

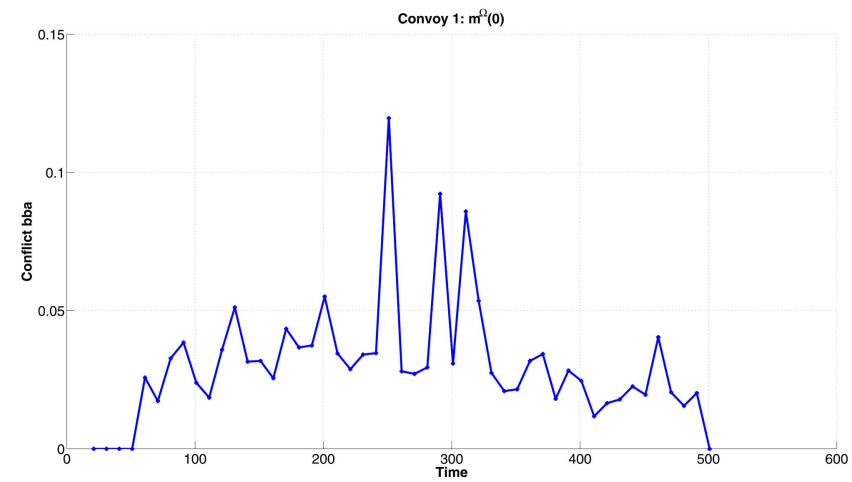

(e)

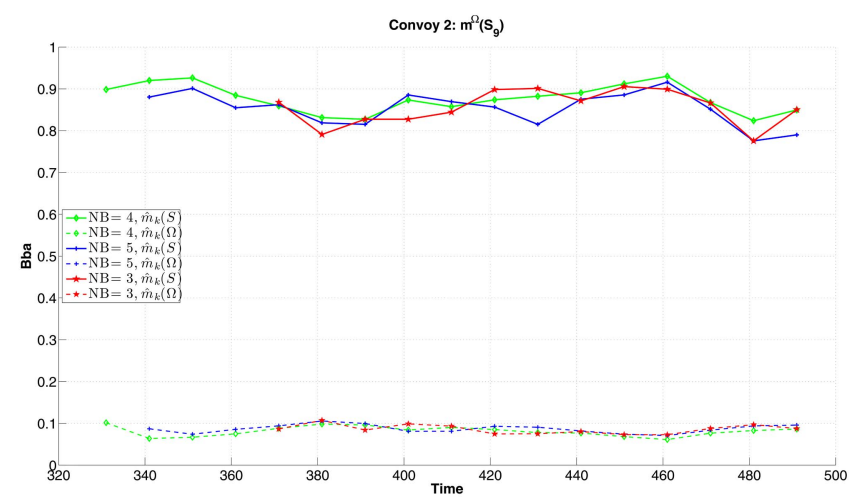

(b)

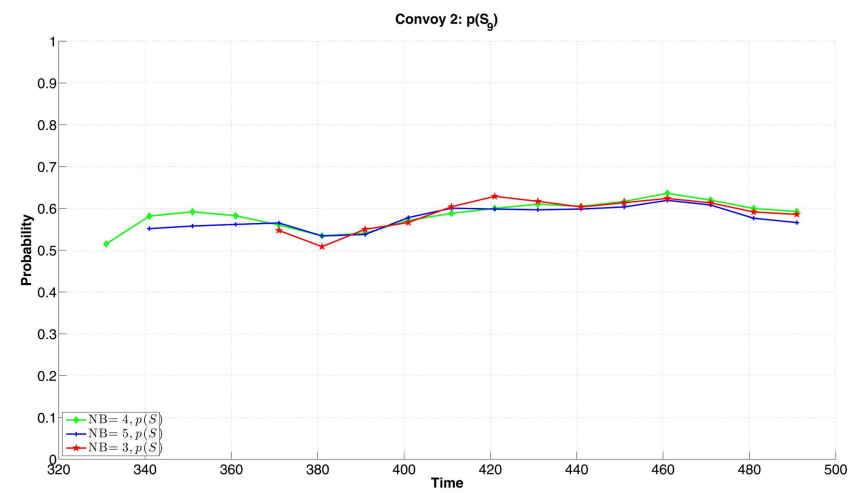

(d)

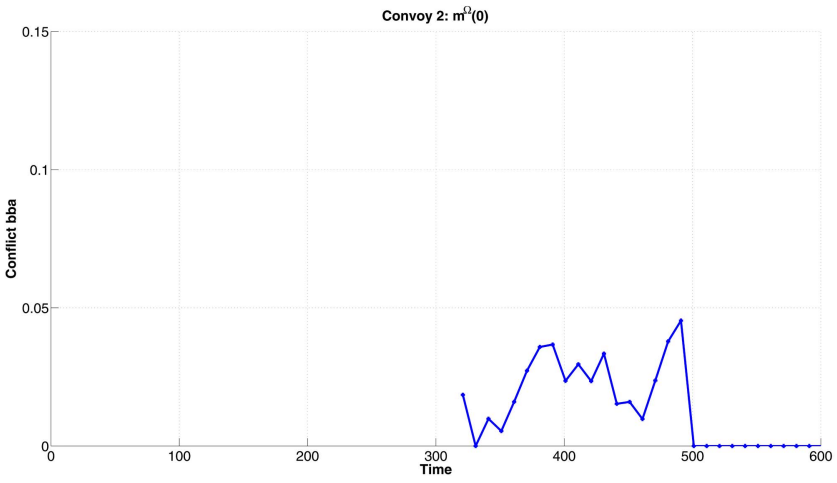

(f)

Fig. 12. Convoy detection and evaluation results for scenario 2. (a) Confidence on 8-then-4 target convoy. (b) Confidence on only-4 target convoy. (c) Probability of having 8-then-4 target convoy. (d) Probability of having only-4 target convoy. (e) Conflict on 8-then-4 target convoy. (f) Conflict on only-4 target convoy.

similar shape for the estimation of the number of targets. They are both closer to the ground truth than the MHT with road constraints. They are more reactive to cardinality changes. Concerning the OSPA distance it is considerably lower for the hybrid algorithm than the MHT algorithm.

3) Convoy Detection and Evaluation Results: During the simulation, two convoys are moving according to the following.

a) The convoy is moving on the main road, composed of eight targets at the begining and four targets at the end. Between time $t=400$ and $t=570 \mathrm{~s}$, the aggregate is composed of nine targets because an additional independent target passes the convoy between time $t=241$ and $t=291 \mathrm{~s}$.

b) The convoy is created after the four last targets turn on the right (from time $t=300 \mathrm{~s}$ ).

Results for convoy detection and evaluation are summarized in Fig. 12. Figures 12(a) and (b) present the credibilistic results compared with the probabilistic results (Figs. 12(c) and (d)). Figures 12(a) and (c) compare results obtained for the 8-then-4 target convoy with results obtained for the only-4 target convoy (Figs. 12(b) and (d)).

Concerning the convoy of 8 targets at the beginning, the probability of having a convoy is quite 
regular over time (Fig. 12(c)), but this probability is quite low (less than 0.6 when eight targets are in the convoy and less than 0.7 when four targets are in the convoy). With the credibilistic approach results are more stable (Fig. 12(a)). The belief of having an 8 -target convoy is close to 0.9 (between time $t=1$ and $t=300 \mathrm{~s}$ ), as well as the belief of having a 4-target convoy (between time $t=320$ and $t=490 \mathrm{~s}$ ). During the passing stage (between time $t=241$ and $t=291$ s), the belief of having 8 targets stays higher than the belief of having 9 targets. At this moment the conflict (Fig. 12(e)) becomes higher.

Concerning the convoy of 4 targets due to the convoy separation, the probability of having a convoy is quite regular over time (Fig. 12(d)), but also quite low. The belief of having a convoy is also quite regular over time but is close to 0.9 all the time. As a consequence the conflict (Fig. 12(f)) stays low (less than 0.05), meaning that no maneuver occurs.

\section{CONCLUSION}

In this article we first proposed an efficient architecture for OID. Inspired by the JDL model, our process uses two levels of granularity in order to detect and evaluate a specific object of interest, which is the convoy. The first level is a sophisticated MTT algorithm, efficient for many situations (large and various number of targets, new birth and death process, closely-spaced targets,...). The second level is based on the modeling of objects of interest with graphical models by using the relationship between their dynamics, type, environment,.... This method has proved its efficiency and robustness on several situations involving convoys. A more elaborate study on the generalization of the convoy detection method to any object of interest would be an interesting topic for further research.

\section{REFERENCES}

[1] Steinberg, A. N. and Bowman, C. L. Rethinking the JDL data fusion levels. Proceedings of the National Symposium on Sensor and Data Fusion, 2004.

[2] Bar-Shalom, Y.

Multitarget-Multisensor Tracking: Principle and Techniques.

Waltham, MA: Academic Press, 1995.

[3] Pollard, E., Pannetier, B., and Rombaut, M. Hybrid algorithms for multitarget tracking using MHT and GM-CPHD.

IEEE Transactions on Aerospace and Electronic Systems, 47, 2 (Apr. 2011), 832-847.

[4] Pollard, E., Pannetier, B., and Rombaut, M. A particle-filtering approach to convoy tracking in the midst of civilian traffic.

Proceedings of SPIE, vol. 6968, Signal Processing, Sensor Fusion, and Target Recognition XVII, Orlando, FL, Mar. 2008.
[5] Pollard, E., Rombaut, M., and Pannetier, B.

Bayesian networks vs. evidential network: An application to convoy detection.

In E. Hüllermeier, R. Kruse, and F. Hoffmann (Eds.),

Proceedings of the 13th International Conference on Information Processing and Management of Uncertainty in Knowledge-Based Systems: Theory and Methods, Part I, Dortmund, Germany, June/July 2010, pp. 31-39.

[6] Bar-Shalom, Y. and Blair, D.

Multitarget-Multisensor Tracking: Applications and Advances, vol. III.

Norwood, MA: Artech House, 2000.

[7] Bar-Shalom, Y. and Fortmann, T.

Tracking and Data Association.

Waltham, MA: Academic Press, 1988.

[8] Blackman, S.

Multiple Target Tracking with Radar Applications.

Norwood, MA: Artech House, 1986.

[9] Goodman, I., Mahler, R., and Nguyen, H. T.

Mathematics of Data Fusion.

Boston, MA: Kluwer Academic Publishers, 1997.

[10] Mahler, R.

A theory of PHD filters of higher order in target number. In I. Kadar (Ed.), Proceedings of SPIE, vol. 6235, Signal Processing, Sensor Fusion, and Target Recognition XV, Orlando, FL, 2006, p. 62350K

[11] Mahler, R.

PHD filters of higher order in target number.

IEEE Transactions on Aerospace and Electronic Systems, 43, 4 (Oct. 2007), 1523-1543.

[12] Mahler, R.

Multitarget Bayes filtering via first-order multitarget moments.

IEEE Transactions on Aerospace and Electronic Systems, 39, 4 (Oct. 2003), 1152-1178.

[13] Vo, B-T., Vo, B-N., and Cantoni, A.

Analytic implementations of the cardinalized probability hypothesis density filter.

IEEE Transactions on Signal Processing, 55, 7 (July 2007), 3553-3567.

[14] Vo, B-N., Singh, S., and Doucet, A.

Sequential Monte Carlo methods for multitarget filtering with random finite sets.

IEEE Transactions on Aerospace and Electronic Systems, 41, 4 (Oct. 2005), 1224-1245.

[15] Lin, L., Bar-Shalom, Y., and Kirubarajan, T. Track and PHD filter for multitarget tracking. IEEE Transactions on Aerospace and Electronic Systems, 42, 3 (July 2006), 778-795.

[16] Clark, D., Panta, K., and Vo, B-N.

The GM-PHD filter multiple target tracker.

Proceedings of the 2006 9th International Conference on Information Fusion, Florence, Italy, July 2006, pp. 1-8.

[17] Erdinc, O., Willett, P., and Bar-Shalom, Y.

The bin-occupancy filter and its connection to the PHD filters.

IEEE Transactions on Signal Processing, 57, 11 (Nov. 2009), 4232-4246.

[18] Panta, K., Clark, D., and Vo, B-N.

Data association and track management for the Gaussian mixture probability hypothesis density filter.

IEEE Transactions on Aerospace and Electronic Systems, 45, 3 (July 2009), 1003-1016.

[19] Sittler, R.

An optimal data association problem in surveillance theory.

IEEE Transactions on Military Electronics, MIL-8, 2

(1964), 125-139. 
Clark, D. and Vo, B.

Convergence analysis of the Gaussian mixture PHD filter. IEEE Transactions on Signal Processing, 55, 4 (Apr. 2007), 1204-1212.

[21] Kirubarajan, T., et al.

Ground target tracking with variable structure IMM estimator.

IEEE Transactions on Aerospace and Electronic Systems, 36, 1 (Jan. 2000), 26-46.

[22] Pannetier, B., Nimier, V., and Rombaut, M.

Multiple ground target tracking with a GMTI sensor.

Proceedings of the 2006 IEEE International Conference on Multisensor Fusion and Integration for Intelligent Systems, Heidelberg, Germany, Sept. 2006, pp. 230-236.

[23] Blackman, S. and Popoli, R.

Design and Analysis of Modern Tracking Systems. Norwood, MA: Artech House, 1999.

[24] Kirubarajan, T. and Bar-Shalom, Y.

Tracking evasive move-stop-move targets with a MTI radar using a VS-IMM estimator.

In O. E. Drummond (Ed.), Proceedings of SPIE, vol. 4048, Signal and Data Processing of Small

Targets, 2000, pp. 236-246. [Online], available: http://link.aip.org/link/?PSI/4048/236/1.

[25] Pannetier, B., Benameur, K., and Nimier, V.

VS-IMM using road map information for a ground target tracking.

Proceedings of the 2005 8th International Conference on Information Fusion, vol. 1, Philadelphia, PA, July 25-28, 2005.

[26] Das, S., Grey, R., and Gonsalves, P.

Situation assessment via Bayesian belief networks.

Proceedings of the 5th International Conference on

Information Fusion, vol. 1, Annapolis, MD, July 8-11, 2002, pp. 664-671.

[27] Denis, N. and Jones, E.

Spatio-temporal pattern detection using dynamic Bayesian networks.

Proceedings of the 42nd IEEE Conference on Decision and Control, vol. 5, Maui, HI, Dec. 2003, pp. 4533-4538.

[28] Mirmoeini, F. and Krishnamurthy, V.

Reconfigurable Bayesian networks for adaptive situation assessment in battlespace.

Proceedings of the 2005 IEEE International Conference on Networking, Sensing and Control, Tucson, AZ, Mar. 2005, pp. 810-815.

[29] Johansson, F. and Falkman, G.

Implementation and integration of a Bayesian network for prediction of tactical intention into a ground target simulator.

Proceedings of the 2006 9th International Conference on Information Fusion, Florence, Italy, July 2006, pp. 1-7.
[30] Smets, P

Data fusion in the transferable belief model.

Proceedings of the Third International Conference on Information Fusion, Paris, July 2000, pp. 10-13.

[31] Denœux, T.

Conjunctive and disjunctive combination of belief functions induced by nondistinct bodies of evidence. Artificial Intelligence, 172, 2-3 (Feb. 2008), 234-264.

[32] Benavoli, A., et al.

An application of evidential networks to threat assessment.

IEEE Transactions on Aerospace and Electronic Systems, 45, 2 (Apr. 2009), 620-639.

[33] Ramasso, E.

Reconnaissance de séquences d'états par le modéle des croyances transférables. Application à l'analyse de vidéo d'athlétisme.

$\mathrm{Ph} . \mathrm{D}$. dissertation, Université Joseph Fourier, France, 2007.

[34] Smets, P.

Belief functions: The disjunctive rule of combination and the generalized Bayesian theorem.

International Journal of Approximate Reasoning, 9, 1 (1993), 1-35.

[35] Pollard, E., Pannetier, B., and Rombaut, M.

Convoy detection processing by using the hybrid algorithm (GMCPHD/VS-IMMC-MHT) and dynamic Bayesian networks.

Proceedings of the 12th International Conference on Information Fusion, Seattle, WA, July 6-9, 2009, pp. 907-914.

[36] Pollard, E., Pannetier, B., and Rombaut, M.

Performances in multitarget tracking for convoy detection over real GMTI data.

Proceedings of the 2010 13th International Conference on Information Fusion, Edinburgh, Scotland, July 2010, pp. $1-7$.

[37] Pollard, E.

Évaluation de situations dynamiques multicibles par fusion de donné es spatio-temporelles.

Ph.D. dissertation, Université de Grenoble, France, 2010.

[38] Schuhmacher, D., Vo, B-T., and Vo, B-N.

A consistent metric for performance evaluation of multi-object filters.

IEEE Transactions on Signal Processing, 56, 8 (Aug. 2008), 3447-3457. 

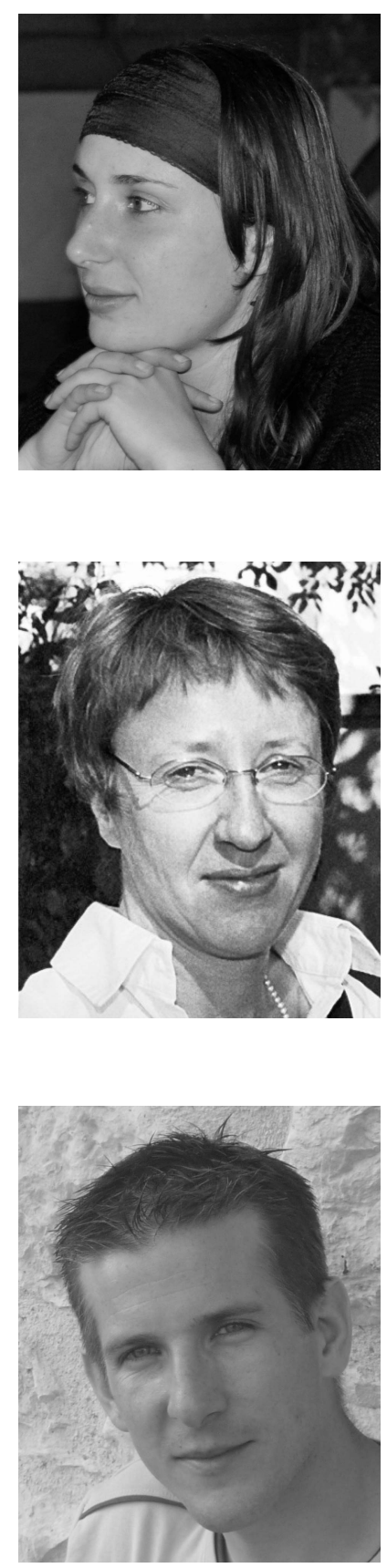

Evangeline Pollard obtained her signal and image processing M.Sc. in 2007 from the University of Lyons. That same year she graduated from the "CPE-Lyons" Engineering School in electronics, telecommunications and computing. In 2010 she obtained her Ph.D. at the French Aerospace Lab (Onera).

During 2005-2006 she worked at the German Aerospace Laboratories (DLR) in Munich on the TerraSAR-X processor. After a postdoc with the Livic and the Université de Sherbrooke, Canada, for driving assistance applications, she is pursuing her work on data fusion and multi-target tracking on driving applications at Inria-Imara, Paris, France.

Michèle Rombaut graduated from the Ecole Universitaire d'Ingénieurs de Lille, France and received her Ph.D. from the University of Lille in electronic systems.

Since 1985 she has been with UTC Compiègne as an associate professor and since 1994 as a professor. Since 2002, at the University Joseph Fourier of Grenoble, she has been conducting her research at GIPSA-Lab (Grenoble Images Speech Signals and Automatics), especially in data fusion and the transferable belief model.

Benjamin Pannetier was born in Paris on November 30th, 1979. He received his B.Sc. in math from the University of Marne la Vallée and his Ph.D. in automatic control and signal processing from the University of Grenoble in 2006.

Since 2005 he has been a research engineer at the French Aerospace Lab (ONERA). His research interests include target tracking, detection/estimation theory, and data fusion for battlefield surveillance systems for the French army. With Professor Rombaut, University of Grenoble, he is working on a new approach for abnormal behavior detection. 